\title{
Synchronous invasive ductal carcinoma and intravascular large B-cell lymphoma of the breast: a case report and review of the literature
}

\author{
Christopher Wei Guang Ho ${ }^{1 *}$, Sangeeta Mantoo ${ }^{2}$, Chin Hong Lim ${ }^{1}$ and Chow Yin Wong ${ }^{1}$
}

\begin{abstract}
Primary breast lymphomas (PBLs) represent less than 1\% of all breast malignancies. Intravascular large B-cell lymphoma (ILBCL) is a rare, aggressive form of extranodal lymphoma. Breast involvement has only been described in the literature once previously. ILBCL is characterized by the proliferation of tumour cells within the lumen of small vessels of involved organs, resulting in their eventual occlusion. Clinical features are often vague, diagnosis is difficult and delayed, and prognosis is usually poor. We report the first ever case of synchronous ILBCL and invasive ductal carcinoma (IDC) of the breast in a patient presenting with pyrexia of unknown origin and altered mental status who underwent modified radical mastectomy and subsequent chemotherapy, and review the literature regarding intravascular large B-cell lymphoma, PBLs and synchronous carcinomas and lymphomas of the breast.
\end{abstract}

Keywords: Intravascular large B-cell lymphoma, Synchronous tumour, Primary breast lymphoma, Breast cancer, Invasive ductal carcinoma

\section{Background}

The breast is one of the least common sites for malignant extranodal lymphomas. Primary breast lymphomas (PBLs) constitute less than $1 \%$ of all breast neoplasms, and are usually non-Hodgkin's lymphomas. A wide variety of histological subtypes have been reported, and it is thought that patients with PBLs have a stage-for-stage prognosis similar to other patients with lymphomas of the same histology that present at other sites [1]. PBLs usually present as a painless, solitary mass, often with associated ipsilateral axillary lymphadenopathy. Multiple masses and diffuse breast enlargement (unilateral or bilateral) have also been reported. Skin retraction, erythema, peau d'orange and nipple involvement are rare. The most frequent mammographic appearance is a well-circumscribed uncalcified mass. Other radiographic features include amorphous or spiculated uncalcified masses and diffusely increased parenchymal density. Ultrasound usually reveals hypoechoic lesions with well-defined borders that lack significant

\footnotetext{
* Correspondence: christopher.ho03@imperial.ac.uk

'Department of General Surgery, Singapore General Hospital, Outram Road,

Singapore 169608, Singapore

Full list of author information is available at the end of the article
}

posterior enhancement or acoustic shadowing that may be confused with a benign cyst [2].

Intravascular large B-cell lymphoma (ILBCL) is a rare, high-grade extranodal diffuse B-cell lymphoma that has only been described in the breast once previously in a patient who had bilateral symmetrical breast engorgement with thickened, peau d'orange skin [3]. The median age at diagnosis is 67 years and it has a male:female sex distribution of 1.3:1. ILBCL may affect virtually any organ and is characterized by the clonal proliferation of malignant lymphocytes within the lumen of small vessels, resulting in their consequent blockage. Parenchyma, lymph nodes and the reticuloendothelial system are relatively spared [4]. In contrast to other lymphomas that present with lymphadenopathy and tumour mass, ILBCLs present heterogeneously depending on the organ affected. Common clinical features include pyrexia of unknown origin, generalized fatigue, cutaneous lesions (including rashes, plaques, nodules, ulcers and hyperpigmentation) and neurological changes (including altered consciousness, sensorimotor deficits, seizures, paresis and dementia). Gastrointestinal symptoms, anaemia, thrombocytopenia, oedema and dyspnoea have also been reported. Due to its wide-ranging, non-specific signs and symptoms, the diagnosis of ILBCL is often difficult 
and treatment consequently delayed. Combined with its aggressive nature, disseminated disease is often present when ILBCL is ultimately recognized, resulting in a dismal prognosis [4].

We describe the first case of synchronous ILBCL and invasive ductal carcinoma (IDC) of the breast, and illustrate the diagnostic pitfalls and therapeutic dilemmas commonly encountered by clinicians when faced with ILBCL and synchronous tumours.

\section{Case presentation}

A 75-year-old Malay woman with a background of diabetes mellitus, hypertension, hyperlipidaemia and endometrial carcinoma who had undergone curative resection and was now in remission presented with a 6-month history of flu-like symptoms and intermittent swinging pyrexia associated with chills and rigors. The patient's family had also noticed that she was becoming increasingly confused and drowsy.

The patient had no gastrointestinal, genitourinary or respiratory symptoms, and did not have signs of meningitis. Physical examination was normal. Extensive infective, malignancy and autoimmune screens did not yield any positive results. Repeated computed tomography (CT) and magnetic resonance imaging (MRI) of the brain were unremarkable. Bone marrow biopsy revealed a hypercellular marrow without any evidence of haematolymphoid malignancy or metastasis and was sent for karyotyping. Bone marrow lymphocytes showed normal morphology and featured a diffuse interstitial infiltrate comprising CD3 positive T-cells with fewer CD20 positive B-cells.
CT of the thorax, abdomen and pelvis was unremarkable except for a $16 \mathrm{~mm}$ lesion in the right inferior breast. Mammography confirmed an irregular $12 \times 11 \times 9 \mathrm{~mm}$ spiculated mass in the 6 o'clock position with ill-defined margins (Figure 1). Fine needle aspiration cytology was suggestive of malignancy, and positron emission tomography (PET)-CT showed a hypermetabolic right breast nodule suspicious for primary breast tumour (Figure 2). No other fluorodeoxyglucose (FDG)-avid lesions were noted.

As extensive investigations had failed to identify the reason for the patient's symptoms, the presumptive diagnosis was that of a breast carcinoma-related paraneoplastic syndrome causing fever and confusion. A modified radical mastectomy with level II axillary clearance was performed, and the fever resolved immediately after surgery. The patient also became more alert, albeit only for the first 2 postoperative days before becoming drowsy again.

Histological analysis confirmed a $20 \mathrm{~mm}$ IDC (grade I, oestrogen receptor (ER) and progesterone receptor (PR) positive, human epidermal growth factor receptor 2 (HER2) negative) with minimal ductal carcinoma in situ. In addition, the small- and medium-sized vessels surrounding the focus of IDC and the breast tissue revealed large atypical intravascular cells (Figure 3A,B,C). These atypical cells were CD20 positive B-lymphocytes with small to moderate amounts of cytoplasm and round to irregular, vesicular and nucleolated nuclei with discernible mitoses (Figure 3D). These large lymphoid cells were strongly and diffusely reactive to MUM-1, and showed weak positivity for CD5, BCL6 and CD10. No expression was seen with cyclin D1, CD23 and CD3. MIB1 proliferative index was more than
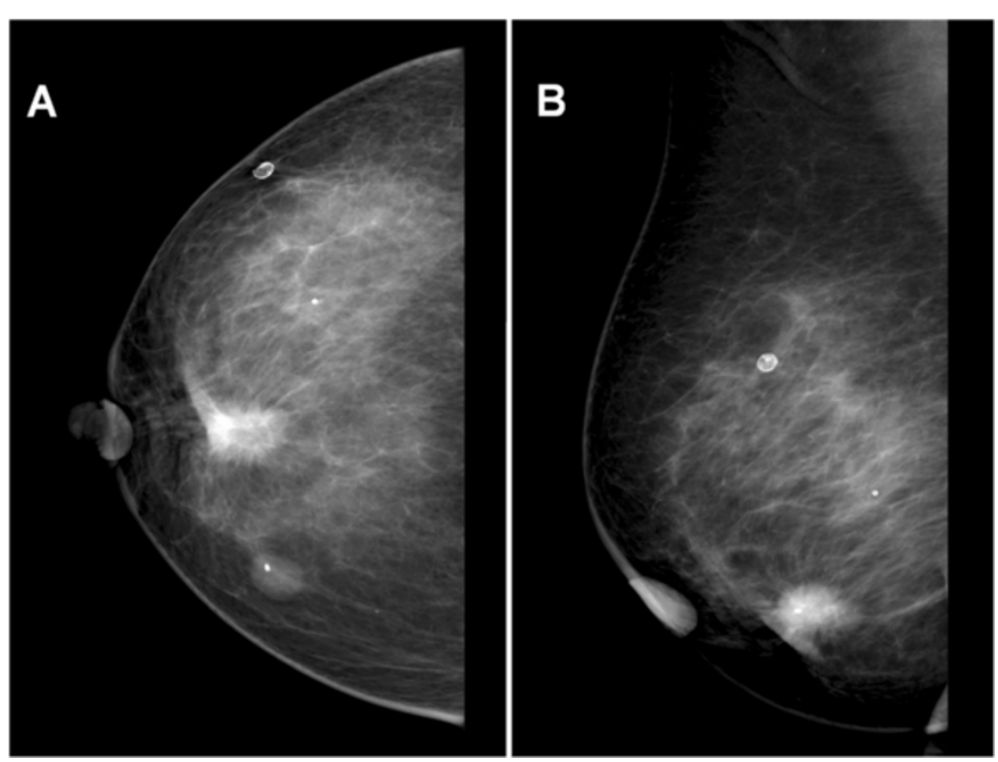

Figure 1 Mammogram. (A) Craniocaudal and (B) mediolateral oblique views of the patient's right breast. There is a 19 mm spiculated mass with associated architectural distortion in the lower central breast highly suspicious for malignancy. There is also a partially calcified fibroadenoma in the lower inner quadrant and a few calcified oil cysts in the upper outer quadrant. 


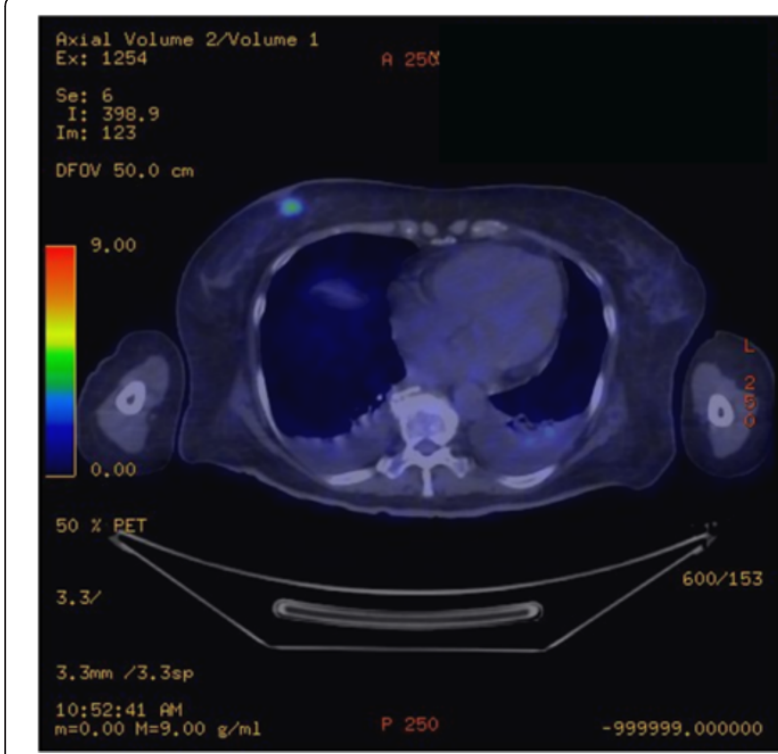

Figure 2 PET-CT. A $1.8 \times 1.4 \mathrm{~cm}$ spiculated mildly FDG-avid right breast nodule just below the level of the nipple is demonstrated (maximum standardized uptake value 3.9). FDG, fluorodeoxyglucose; PET-CT, positron emission tomography-computed tomography.
$90 \%$. Bone marrow karyotyping also revealed that 2 out of 20 metaphases analyzed had complex structural abnormalities, loss of $\mathrm{X}$ chromosome and trisomy 18 . Thus, a unique diagnosis of synchronous IDC and non-germinal centre type ILBCL was made.

The patient was started on the rituximab, cyclophosphamide, hydroxydaunorubicin, Oncovin (vincristine) and prednisolone (R-CHOP) chemotherapy regimen which led to a sustained improvement in her mental state. Unfortunately, the subsequent tumour lysis resulted in acute renal failure requiring haemodialysis. Finally, the patient developed neutropenic sepsis, and passed on 2 weeks after commencing chemotherapy.

\section{Discussion}

This case represented a significant diagnostic challenge because of the patient's non-specific clinical features, the presence of a confounding synchronous IDC and the rarity of ILBCL, especially in the breast. Similar to the previous reported case of ILBCL involving the breast [3], this patient also satisfied the diagnostic criteria for primary breast lymphomas, but did not have any signs or symptoms localizing to the breast. One of the differentials entertained was a paraneoplastic syndrome, which results from tumour secretion of substances that mimic the effects of endogenous hormones and cytokines, or from the

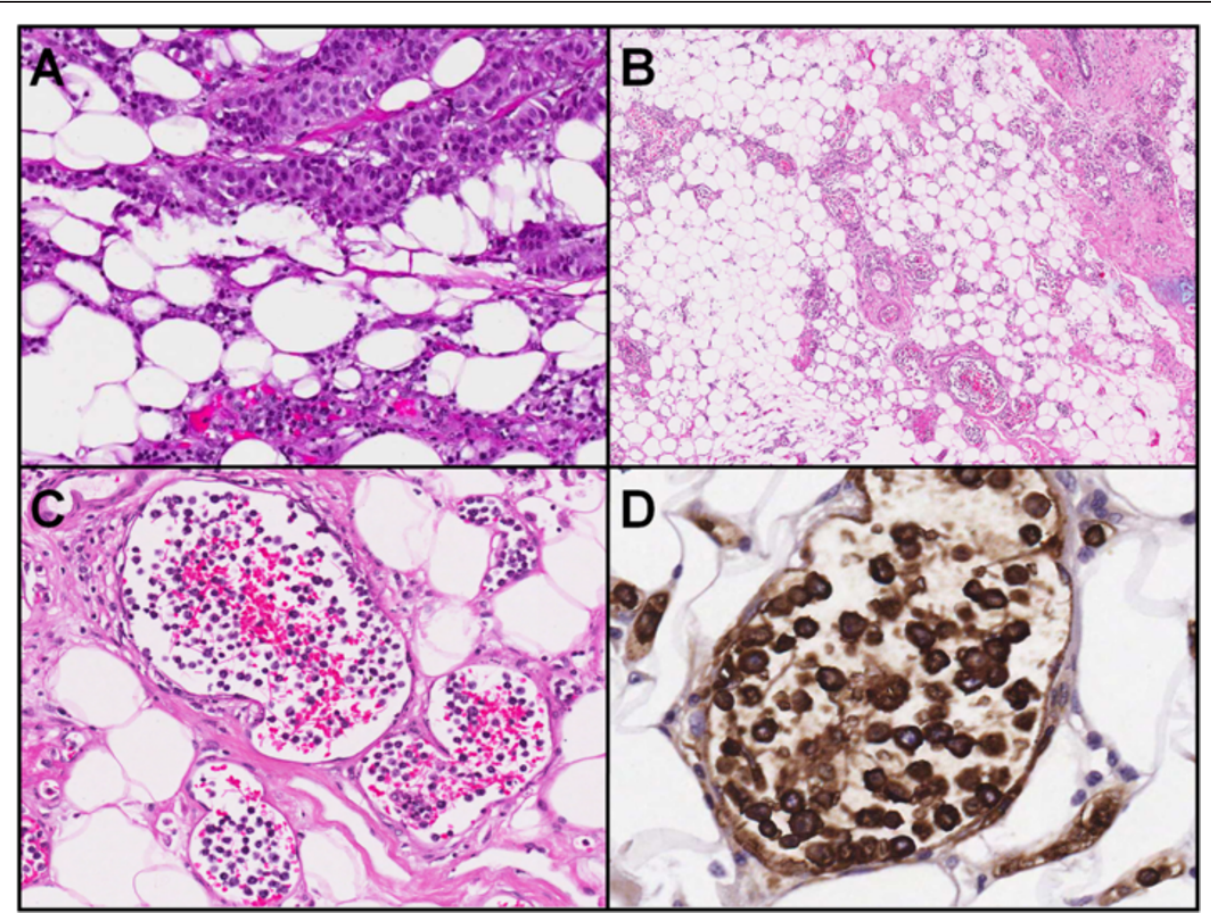

Figure 3 Histological analysis. (A) IDC (top right) with ILBCL (bottom centre) 130x magnification. (B) ILBCL in the surrounding breast tissue away from the IDC. Most of the blood vessels show darkly stained atypical lymphoid cells within their lumen 40x magnification. (C) Atypical lymphoid cells within the blood vessels 200x magnification. (D) CD20 reactive atypical large cells within the blood vessels 400x magnification. IDC, invasive ductal carcinoma; ILBCL, intravascular large B-cell lymphoma. 
production of antitumour antibodies that cross-react with normal cells. Paraneoplastic syndromes involving the breast are rare, and it is estimated that only $36 \%$ of such patients have identifiable paraneoplastic antibodies [5]. Fever is one of the most common features, and is thought to be cytokine-mediated. In addition, specific onconeural autoantibodies such as anti-Ri, anti-Ro and anti-Yo, which may form in response to the breast neoplasm, may also target tissue antigens of the central and peripheral nervous system $[5,6]$. However, in contrast to this case, the neurological manifestations described were motor, sensory and visual with no impairment of consciousness.

Given its rarity and protean manifestations, the diagnosis of ILBCL is one of considerable difficulty. Nevertheless, ILBCL often presents with pyrexia of unknown origin, as well as cutaneous and neurological features [4]. Neuroradiological signs, however, are present in only half of patients with neurological symptoms; CT is frequently normal in patients with neurological manifestations of ILBCL and is therefore generally considered non-diagnostic, and MRI is often normal or shows non-specific hyperintense white matter lesions suggestive of small vessel ischaemic disease or demyelination [7]. As illustrated in this case, PET-CT may also fail to demonstrate cerebral localization of ILBCL. Such false negatives have also been reported in other studies in patients with neurological symptoms [4,8]. Bearing this in mind, a low threshold for biopsy of suspicious lesions should be maintained in the appropriate clinical context, as histological diagnosis is paramount to the timely and appropriate treatment vital to improving patient outcome. The classic appearance and immunophenotype of ILBCL is that of large malignant CD20, CD19, CD10, CD5 and CD79a positive B-lymphocytes filling small vascular lumens. In addition, chromosomal analysis may reveal abnormalities in 1p and trisomy 18 [4,9].

Numerous theories attempt to explain the pathogenesis of synchronous carcinoma and lymphoma of the breast. Patients with lymphoma are known to be chronically immunosuppressed, which may predispose to the development of a second malignancy [10]. It has also been suggested that the antigenic stimulation from an as yet undefined breast carcinoma antigen may drive the development of a mucosa-associated lymphoid tissue (MALT) lymphoma, analogous to the effect of chronic Helicobacter pylori infection on the pathogenesis of gastric MALT lymphomas [11]. Another postulated mechanism is that both tumours share the same aetiological factors, with mutation in the ataxia telangiectasia mutated (ATM) tumour suppressor gene [12], as well as infection with the Epstein-Barr virus (EBV) [13] and mouse mammary tumour virus (MMTV) [14] all currently implicated.

Mastectomy has traditionally been considered the gold standard treatment for PBLs, however, this has now shown to offer no survival benefit or protection from recurrence
[2]. Chemotherapy, typically an anthracycline-based regime with or without rituximab has emerged as the mainstay of treatment, and can be combined with radiotherapy in patients with intermediate or high grade lymphomas $[1,2,15]$. There are no randomized controlled trials comparing treatment for patients with ILBCL, however, as dissemination is common at diagnosis, aggressive systemic chemotherapy is usually warranted. Methotrexate, either intrathecal or high-dose systemic, should be incorporated if central nervous system involvement is present [16]. The addition of rituximab has been shown to significantly improve clinical outcomes [4,17], and autologous stem cell transplantation has been shown to be effective in young patients with good performance status [17]. Because of the rarity of synchronous carcinoma and lymphoma of the breast, there is no consensus on treatment in such cases and it remains uncertain if such synchronous tumours should be viewed as two distinct clinical entities with corresponding separate treatments, or as a single disease with therapy that encompasses both tumour types.

This unique synchronous tumour comprising of an IDC and ILBCL in a patient presenting with pyrexia of unknown origin and altered mental status highlights the difficulties in diagnosing ILBCLs as well as synchronous tumours in general. Although the optimal management for such synchronous tumours is still a matter for further studies, adequate histological assessment is vital to ensure appropriate treatment. Finally, additional investigation into the pathogenesis of synchronous carcinoma and lymphoma of the breast may lead to novel preventative and therapeutic strategies for such synchronous tumours as well as for its individual constituent neoplasms.

\section{Conclusion}

We report the first case of synchronous carcinoma and ILBCL of the breast. ILBCL is rare, and has only been described in the breast once previously. Its clinical spectrum is broad, and a high degree of suspicion is required to seek early biopsy to establish histological diagnosis. Timely, aggressive, systemic chemotherapy has been shown to significantly improve outcomes. The development and treatment of synchronous breast carcinomas and lymphomas remains uncertain, and further studies should be encouraged.

\section{Consent}

Written informed consent was obtained from the patient for publication of this case report. A copy of the written consent is available for review by the Editor-in-Chief of this journal.

\section{Abbreviations}

ATM: Ataxia telangiectasia mutated; CT: Computed tomography; EBV: Epstein-Barr virus; ER: Oestrogen receptor; FDG: Fluorodeoxyglucose; HER2: Human epidermal growth factor receptor 2; IDC: Invasive ductal carcinoma; ILBCL: Intravascular large B-cell lymphoma; MALT: Mucosa-associated lymphoid tissue; MMTV: Mouse 
mammary tumour virus; MRI: Magnetic resonance imaging; PBL: Primary breast lymphoma; PET: Positron emission tomography; PR: Progesterone receptor; R-CHOP: Rituximab cyclophosphamide, hydroxydaunorubicin, Oncovin, prednisolone.

\section{Competing interests}

The authors declare that they have no competing interests.

\section{Authors' contributions}

$\mathrm{CWGH}, \mathrm{CHL}$ and $\mathrm{CYW}$ treated the patient and conceived the idea. SM performed the pathological analysis and provided the histology slides. CWGH performed the literature search and wrote the manuscript. CYW reviewed and revised the manuscript. All authors read and approved the final manuscript.

\section{Author details}

'Department of General Surgery, Singapore General Hospital, Outram Road, Singapore 169608, Singapore. ${ }^{2}$ Department of Pathology, Singapore General Hospital, Outram Road, Singapore 169608, Singapore.

Received: 1 October 2013 Accepted: 30 March 2014

Published: 8 April 2014

\section{References}

1. Domchek SM, Hecht JL, Fleming MD, Pinkus GS, Canellos GP: Lymphomas of the breast: primary and secondary involvement. Cancer 2002, 94:6-13.

2. Jennings WC, Baker RS, Murray SS, Howard CA, Parker DE, Peabody LF, Vice HM, Sheehan WW, Broughan TA: Primary breast lymphoma: the role of mastectomy and the importance of lymph node status. Ann Surg 2007, 245:784-789.

3. Monteiro M, Duarte I, Cabecadas J, Orvalho ML: Intravascular large B-cell lymphoma of the breast. Breast 2005, 14:75-78.

4. Shimada K, Kinoshita T, Naoe T, Nakamura S: Presentation and management of intravascular large B-cell lymphoma. Lancet Oncol 2009 10:895-902.

5. Altaha R, Abraham J: Paraneoplastic neurologic syndrome associated with occult breast cancer: a case report and review of literature. Breast J 2003, 9:417-419.

6. Gatti G, Simsek S, Kurne A, Zurrida S, Naninato P, Veronesi P, Frasson A, Millen E, Rososchansky J, Luini A: Paraneoplastic neurological disorders in breast cancer. Breast 2003, 12:203-207.

7. Song DK, Boulis NM, McKeever PE, Quint DJ: Angiotropic large cell lymphoma with imaging characteristics of CNS vasculitis. Am J Neuroradiol 2002, 23:239-242.

8. Kawai N, Okada M, Haba R, Yamamoto Y, Tamiya T: Insufficiency of positron emission tomography and magnetic resonance spectroscopy in the diagnosis of intravascular lymphoma of the central nervous system. Case Rep Oncol 2012, 5:339-346.

9. Zuckerman D, Seliem R, Hochberg E: Intravascular lymphoma: the oncologist's "great imitator". Oncologist 2006, 11:496-502.

10. Cuff KE, Dettrick AJ, Chern B: Synchronous breast cancer and lymphoma: a case series and a review of the literature. J Clin Pathol 2010, 63:555-557.

11. Susnik B, Jordi Rowe J, Redlich PN, Chitambar C, Chang CC, Kampalath B: A unique collision tumor in breast: invasive ductal carcinoma and mucosaassociated lymphoid tissue lymphoma. Arch Pathol Lab Med 2004, 128:99-101.

12. Concannon P: ATM heterozygosity and cancer risk. Nat Genet 2002, 32:89-90.

13. Wiernik PH, Etkind PR: The association between breast cancer and lymphoma. Clin Adv Hematol Oncol 2005, 3:695-696.

14. Etkind PR, Stewart AFR, Dorai T, Purcell DJ, Wiernik PH: Clonal isolation of different strains of mouse mammary tumor virus-like DNA sequences from both the breast tumors and non-Hodgkin's lymphomas of individual patients diagnosed with both malignancies. Clin Cancer Res 2004, 10:5656-5664

15. Avenia N, Sanguinetti A, Cirocchi R, Bistoni G, Trastulli S, D'Ajello F, Barberini F, Cavallaro G, Rulli A, Sidoni A, Noya G, De Toma G, Sciannameo F: Primary breast lymphomas: a multicentric experience. World J Surg Oncol 2010, 8:53.
16. Ponzoni M, Ferreri AJ, Campo E, Facchetti F, Mazzucchelli L, Yoshino T, Murase T, Pileri SA, Doglioni C, Zucca E, Cavalli F, Nakamura S: Definition, diagnosis, and management of intravascular large B-cell lymphoma: proposals and perspectives from an international consensus meeting J Clin Oncol 2007, 25:3168-3173.

17. Shimada K, Matsue K, Yamamoto K, Murase T, Ichikawa N, Okamoto M, Niitsu N, Kosugi H, Tsukamoto N, Miwa H: Retrospective analysis of intravascular large B-cell lymphoma treated with rituximab-containing chemotherapy as reported by the IVL study group in Japan. J Clin Oncol 2008, 26:3189-3195.

doi:10.1186/1477-7819-12-88

Cite this article as: Ho et al:: Synchronous invasive ductal carcinoma and intravascular large B-cell lymphoma of the breast: a case report and review of the literature. World Journal of Surgical Oncology 2014 12:88.

\section{Submit your next manuscript to BioMed Central and take full advantage of:}

- Convenient online submission

- Thorough peer review

- No space constraints or color figure charges

- Immediate publication on acceptance

- Inclusion in PubMed, CAS, Scopus and Google Scholar

- Research which is freely available for redistribution

Submit your manuscript at www.biomedcentral.com/submit
C Biomed Central 